\title{
USING COMPUTED TOMOGRAPHY DATA FOR FINITE ELEMENT MODELS OF WOOD BOARDS
}

\author{
Johannes A. J. HUBER ${ }^{* 1}$, Mats EKEVAD ${ }^{2}$ and Olof BROMAN ${ }^{3}$ \\ ${ }^{1}$ Luleå University of Technology \\ Forskargatan 1, 93187 Skellefteå, Sweden \\ johannes.huber@ltu.se \\ ${ }^{2}$ Luleå University of Technology \\ Forskargatan 1, 93187 Skellefteå, Sweden \\ mats.ekevad@1tu.se \\ ${ }^{3}$ Luleå University of Technology \\ Forskargatan 1, 93187 Skellefteå, Sweden \\ olof.broman@1tu.se
}

Key words: CT scanning, Fibre deviation, Gradient structure tensor, Strength grading, Sawn timber

\begin{abstract}
A procedure is presented to generate 3D FE models of timber boards based on CT scans. The boards were tested in four-point bending tests until failure and the local displacement in the pure bending zone was recorded. The $\mathrm{CT}$ scans were treated as 3D images and image processing methods were used to reconstruct the board, the knots and the pith. A new procedure to reconstruct the fibre deviations around knots by accounting for image gradient information was used. A quadratic tetrahedral mesh was generated for the region of the board which was under pure bending in the tests. The fibre directions and the stiffness tensor, scaled by the local density, were transferred into each integration point of the mesh and the bending test was replicated. Preliminary results show that the procedure is able to realistically predict the observed local stiffness of the boards. Further development of the procedure is required to account for dead knots and to extend the procedure for indicating strength and predicting failure.
\end{abstract}

\section{INTRODUCTION}

Strength-graded timber boards with predictable mechanical properties are a prerequisite for engineered wood products used in construction of tall wood buildings, e.g. cross-laminated timber panels or glue laminated timber beams. In sawmills today, strength grading is usually performed heuristically in an automated fashion using established correlation relationships between so-called indicating properties and the strength class of the board [1]. In Sweden, timber strength classes are derived from a fourpoint bending test [2]. The indicating properties are calculated from various measurements at the board level, e.g. flatwise bending, eigenfrequency measurement, visual, laser or X-ray scanning [3, 4]. The available grading methods do not derive or impose any mechanical model from the collected data and are thus bound to accommodate the unique variations of each individual board by statistical relationships. Combined with the fact that the lower 5th-percentile value is used to determine the strength of a board in 
a population of strength-graded boards, current strength-grading methods only exploit a small fraction of the potential strength of individual boards [1]. Improved strength prediction could increase the efficient use of timber and facilitate the production of high-strength timber.

Computed tomography (CT) scanning is known to accurately measure the density inside wooden logs [5]. Recently, some sawmills have adapted CT scanners at the beginning of the saw line to determine the optimal log rotation before sawing. The abundant data generated by the CT scans of the log is today not used for any further processing after the log enters the sawmill. CT data consists of single images (slices) of the scanned object, which together comprise a 3D image consisting of voxels, i.e. pixels with a third spatial dimension, whose size is contingent to the scanner resolution. Each voxel is assigned a scalar intensity value corresponding to the measured density and can be visualised as a greyscale value. Previous research has focused on applying various image analysis methods to detect and reconstruct internal features of the log from CT scans, e.g. the location of the pith, knots, pitch pockets, heartwood, sapwood and annual rings structures [6-9].

Wood is an inhomogeneous and anisotropic material with properties subject to natural variation. During growth, the wood cells in a tree subdivide from the pith radially outwards ( $\mathrm{R}$ direction) and cells from the same growth cycle neighbour each other tangentially (T direction). In longitudinal (L) direction, the cells constitute fibres which are aligned with the growth direction of the tree in the undisturbed zones, i.e. clearwood. Knots result from the growth of branches out of the tree trunk. In the vicinity of knots, the direction of the fibres are severely deviated. In temperate climate zones, the thickness of the cell walls, and consequently the local material density, varies during the seasons which results in visible

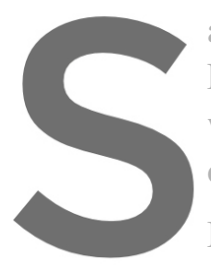
annual rings. In earlyw For wood, high density with lower stiffness and originating in the vicinity Mechanical modelling of individu
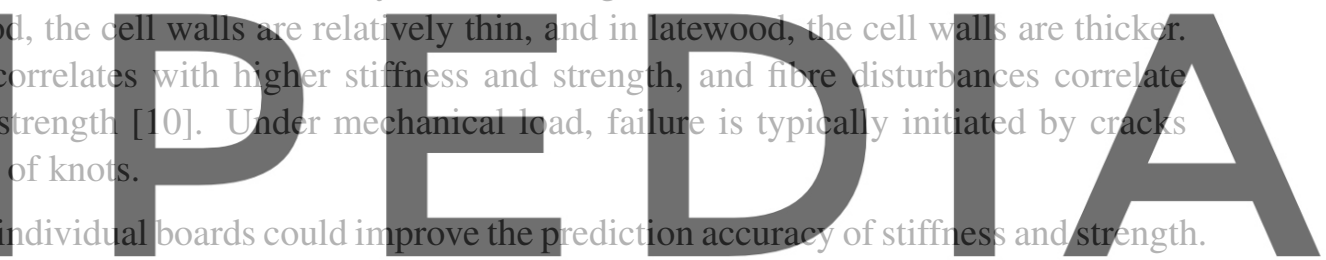

Applying the finite element (FE) method requires detailed information about the location of knots, the

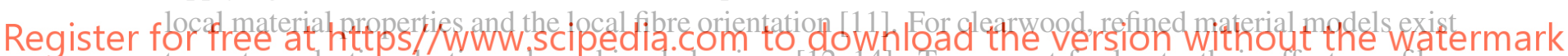
to capture elastic, plastic and cracking behaviour [12-14]. To account for knots, their effects on fibre deviation needs to be reconstructed. So far, reconstructions have been based on optical data from the surface of boards [15-18]. Attempts for using volumetric data, e.g. CT scans, for reconstruction and FE models to predict stiffness and strength have been scarce in the literature.

The goal of the present work is to develop a procedure based on CT scans to

- reconstruct the internal structure and local material properties of wood boards,

- generate 3D FE models, and

- predict the bending stiffness and strength.

This paper presents the current state of this approach and some preliminary results. The developed procedure could be adapted to model also virtual boards extracted from CT scans of logs before sawing. The results of such models could increase the accuracy of stiffness and strength prediction of individual boards at the end of the sawing process. 


\section{MATERIALS AND METHOD}

\subsection{Experiments}

The studied material comprised 20 Norway spruce (picea abies) centre boards (sawn through the pith) at $12 \%$ moisture content (MC) with nominal dimensions of $50 \mathrm{~mm} \times 100 \mathrm{~mm}$ and varying lengths (3.4$5.6 \mathrm{~m})$. The boards were scanned in batches of four using a laboratory medical CT scanner of the model Siemens Somatom Emotion Duo, operated at an acceleration voltage of $110 \mathrm{kV}$ at the facilities of Luleå University of Technology. The scanner recorded a $512 \times 512$ pixel tomography image every $10 \mathrm{~mm}$ along the board length. The resolution in the scanning plane was $0.68 \mathrm{~mm} \times 0.68 \mathrm{~mm}$ and the scanned CT stacks were saved in the medical image format DICOM. In a stack, the point-wise measured intensity values are stored in the dimensionless Hounsfield unit (HU), which is derived from the attenuation of the $\mathrm{X}$-rays in the material.

Each board was loaded until failure in a four-point bending test according to [2]. The global displacement was measured at mid-span between the external supports which were $3 a=1800 \mathrm{~mm}$ apart. In the pure bending zone, i.e. the length section of $a$ centred between the supports, the local relative mid-span displacement $\delta$ was recorded by a measurement gauge. The gauge was fixed to two points, each distanced $250 \mathrm{~mm}$ from the mid-span location. The total force $F$ during the tests was recorded, and for calibration of the models, the moment in the pure bending zone of the test was calculated as in equation 1 , where $M$ is in $\mathrm{Nm}, F$ in $\mathrm{kN}$ and $a$ in $\mathrm{mm}$.

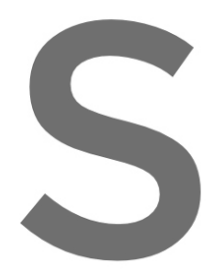

2.2 Board and feature

To analyse the data and prepare it for an FE simulat language Julia [20]. A linear conversion law, supp
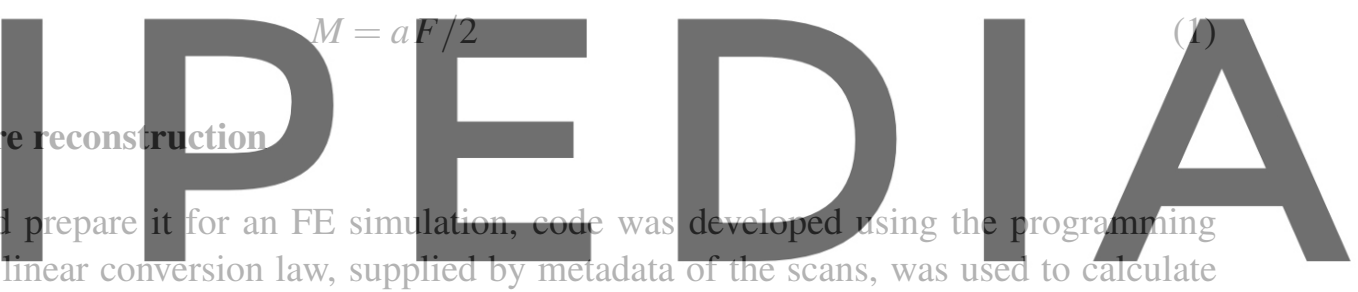

the density in $\mathrm{kg} / \mathrm{m}^{3}$ from HU values of each voxel of the stack of CT slices of a single scan. A prismatic

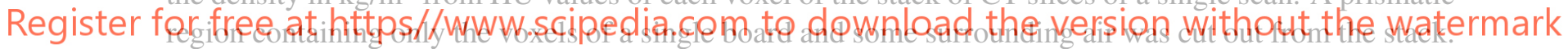

To receive an equal resolution in all dimensions, the values between the slices in longitudinal direction

were linearly interpolated and resampled at $0.68 \mathrm{~mm}$. The data was reduced to the longitudinal section corresponding to the pure bending zone in the four-point bending tests. This reduced dataset can be regarded as a single-channel 3D image carrying density values at discrete locations (voxels) and it is henceforth referred to as $I_{0}(x, y, z)$, where $x$ and $y$ lie in the cross-sectional plane and $z$ is aligned with the length direction of the board (see Figure 1a and 1b).

For segmentation of $I_{0}$ into wood and air, a thresholding rule with $275 \mathrm{~kg} / \mathrm{m}^{3}$ was applied. Voxels with values above this threshold were regarded as part of the board. To reconstruct the pith, first an edge detection [21] was performed on each cross-sectional slice $I_{0}(x, y)$ with $z$ fixed, yielding a binary edge image $B_{i}(x, y)$, where primarily the annual rings were detected as edges. Subsequently, the Hough transform for circles [22] was applied on $B_{i}(x, y)$ to detect the most likely centre of the concentric annual rings. The detected centre was stored as the pith coordinate for each slice and local deviations among the slices were corrected by analysing a sliding window over a length of six slices and setting any outlier pith coordinates to the mean of the coordinates in the window. The collection of pith coordinates in the slices represented the detected pith of the board (see Figure 1a). 


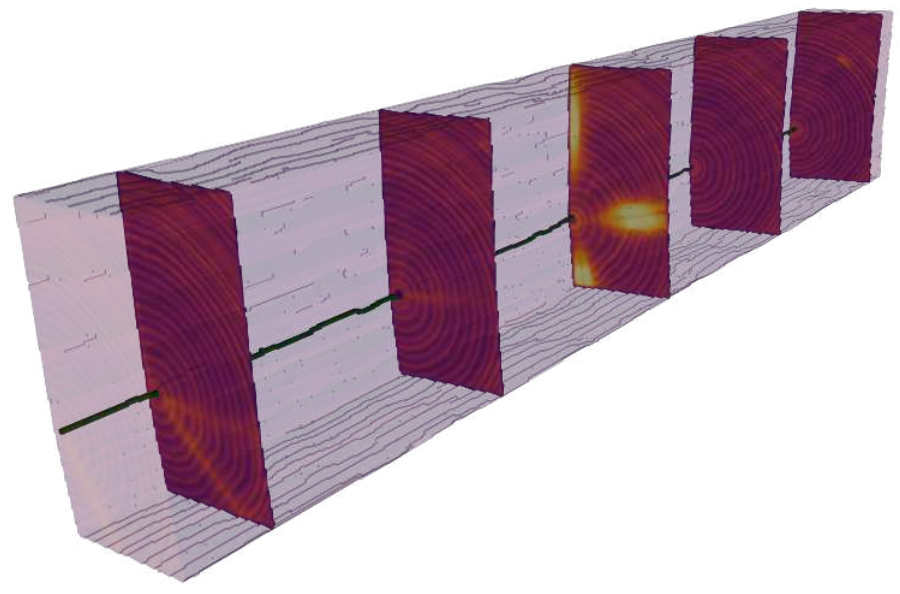

(a) Volume with some slices and the pith

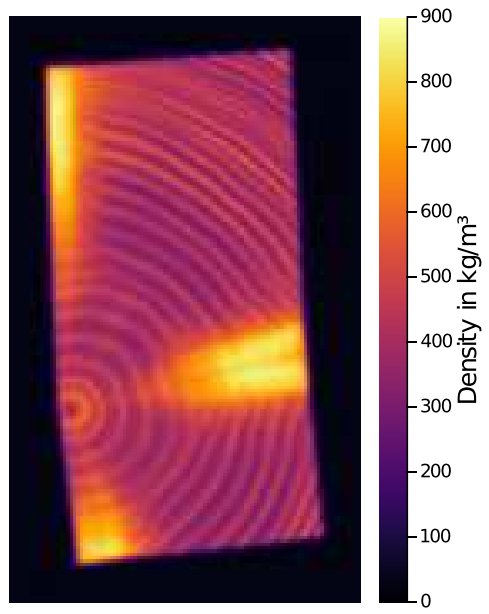

(b) Single slice

Figure 1: Density in a reconstructed board section

To reconstruct the knots, first the voxels belonging to candidates for knots were found in $I_{0}$ by thresholding values above $700 \mathrm{~kg} / \mathrm{m}^{3}$. Each contingent set of voxels above this threshold was assumed to be a knot candidate. For each candidate $k$, its centre of gravity $\mathbf{k}_{g}$ and its first principal axis of inertia $\mathbf{k}_{I}$ was calculated. The closest distance ${ }_{k}$, between the pith and a line passing through $\mathbf{k}_{g}$ with direction $\mathbf{k}_{\text {, }}$

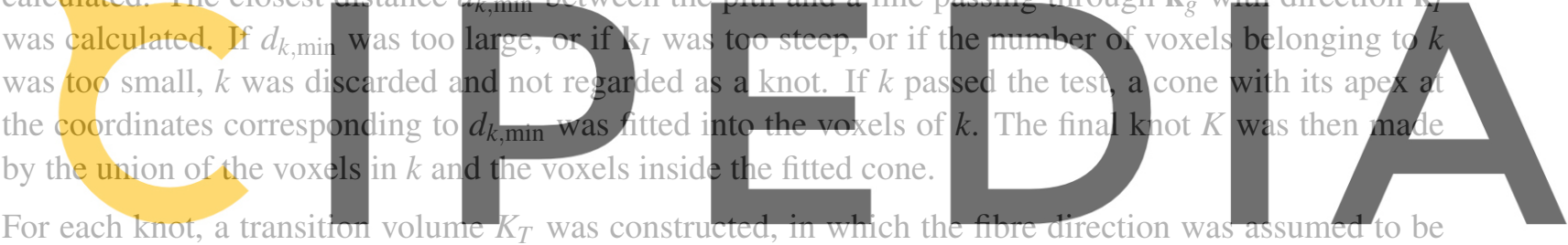
disturbed by the presence of the knot, as proposed in [18]. $K_{T}$ was made by the intersection of two cones:

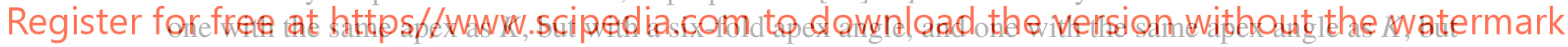
with its apex shifted along $\mathbf{k}_{I}$ such that at least a thickness of 2.5 times the largest knot diameter could be achieved relative to the inner cone of $K$. Figure 2 shows a reconstructed knot and transition volume in relation to the pith.

\subsection{Fibre reconstruction}

An orthotropic material law of wood is based on the local L-R-T coordinate system [10]. Since the stiffness and strength for the $\mathrm{L}$ direction are between 10 and 20 times higher than for $\mathrm{R}$ and $\mathrm{T}$, it is most important to account for the deviations of $\mathrm{L}$, in specific around knots. Common fibre reconstruction approaches $[23,24]$ exploit the similarity between the lateral fibre deviations around a knot and a laminar fluid flow around a disturbance. Alternatively, the lateral deviations can be modelled by finding the maximum principal stresses around a knot under load and aligning the $\mathrm{L}$ direction with the principal directions in an iterative process [11]. To account for the fibre deviations (diving angle) in a slice along the L-R plane through a knot, polynomial functions of the distance to the knot can be used [23, 25]. In contrast to existing FE modelling approaches of boards where primarily surface data has been used [17, 


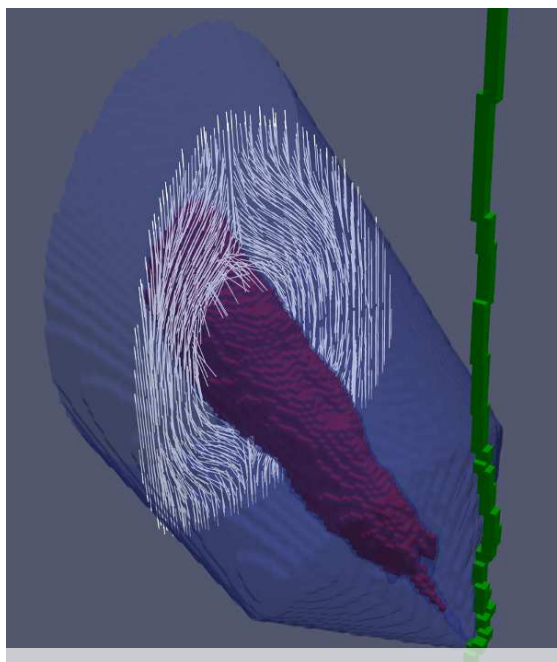

Figure 2: Transition volume with a slice of the reconstructed $\mathrm{L}$ direction

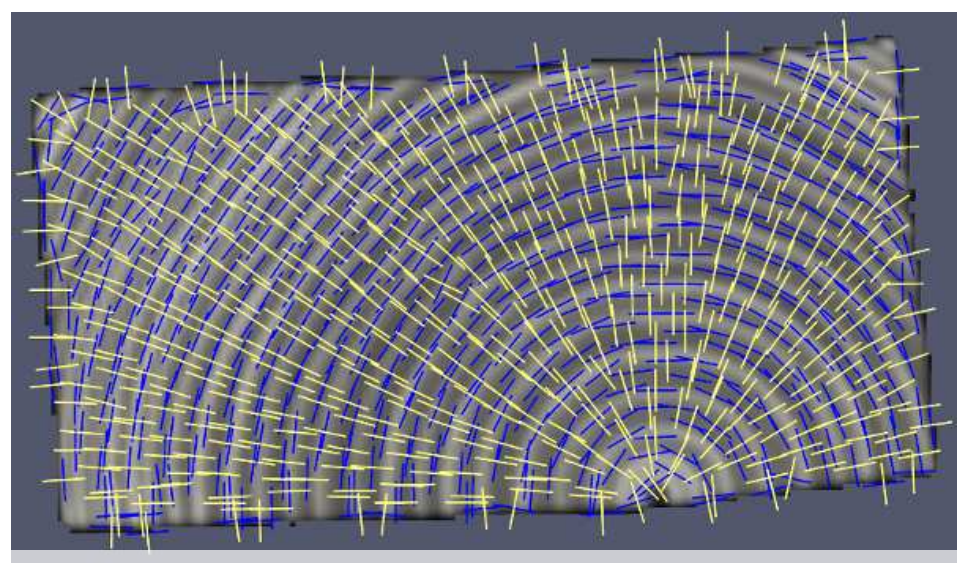

Figure 3: Reconstructed R (yellow) and T (blue) directions in a clearwood slice

18], the present study uses 3D image data and the procedure herein to reconstruct the fibre orientation is primarily based on common image analysis and processing methods.

An essential tool in image processing is convolution, which performs an averaging scheme over the

neighbourhood of each pix kernel $w(x, y)$ is applied
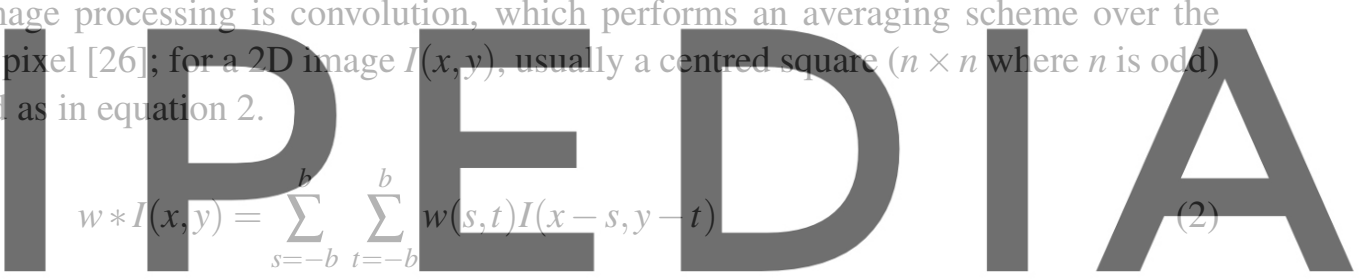

where $b$ is the distance from the centre of the kernel in pixels. At the image edges, various extension

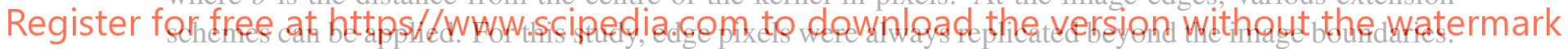

The reconstruction of the fibre directions was based primarily on the gradient structure tensor (GST), which can be used to find local orientations of structures in images of any dimension [27]. Example use cases of the GST include the reconstruction of the local R and $\mathrm{T}$ direction in cross-sectional slices of wood pieces [28], and the 3D fibre orientation in fibre-reinforced concrete [29]. For this study, the 2D GST was applied to suitable slices of $I_{0}$ by proceeding as follows.

In the first step, a smoothed image slice $I_{S}$ was calculated as in equation 3 by using a Gaussian kernel $w_{\sigma_{1}}$ with standard deviation $\sigma_{1}=1$ to reduce noise [26].

$$
I_{s}(x, y)=w_{\sigma_{1}} * I_{0}(x, y)
$$

In the second step, the gradient of $I_{S}$ was calculated. The partial derivative of an image along one of the image axes can be approximated by a finite difference scheme towards the neighbours of each pixel. This is usually accomplished by a $3 \times 3$ derivative convolution kernel for each direction [26], $w_{\Delta x}$ and $w_{\Delta y}$. Here, Bickley kernels have been used $[30,31]$ and the respective gradients $I_{s_{x}}$ and $I_{s_{y}}$ have been calculated as in equations 4 and 5 . 


$$
\begin{aligned}
& I_{S_{x}}(x, y)=\frac{\partial}{\partial x} I_{s}(x, y) \approx w_{\Delta x} * I_{s}(x, y) \\
& I_{S_{y}}(x, y)=\frac{\partial}{\partial y} I_{s}(x, y) \approx w_{\Delta y} * I_{s}(x, y)
\end{aligned}
$$

In the third step, a symmetric second-moment matrix was created from $I_{s_{x}}$ and $I_{s_{y}}$, to avoid cancelling effects of oppositely oriented gradients, and each entry was smoothed by a Gaussian kernel $w_{\sigma_{2}}$ with standard deviation $\sigma_{2}=1.5$ to receive the $\mathbf{G S T}_{I_{s}}(x, y)$ as in equation 6 .

$$
\mathbf{G S T}_{I_{s}}(x, y)=\left[\begin{array}{ll}
w_{\sigma_{2}} * I_{S_{x}}^{2} & w_{\sigma_{2}} *\left(I_{S_{x}} I_{S_{y}}\right) \\
w_{\sigma_{2}} *\left(I_{S_{x}} I_{S_{y}}\right) & w_{\sigma_{2}} * I_{s_{y}}^{2}
\end{array}\right]
$$

In the last step, the eigenvalues $\lambda_{1}, \lambda_{2}$, with $\lambda_{1} \geq \lambda_{2}$, and eigenvectors $e_{1}, e_{2}$ were extracted for $\mathbf{G S T}_{I_{s}}$ and the acceptance criterion in equation 7 was used to check if a local orientation was sufficiently prominent for the respective pixel. If $\lambda_{1}=\lambda_{2}=0$, the criterion was set to 0 . If the criterion was passed, $e_{1}$ and $e_{2}$ were assumed to represent the local material orientations of the respective pixel.

$$
\frac{\left(\lambda_{1}-\lambda_{2}\right)^{2}}{\lambda_{1}^{2}+\lambda_{2}^{2}} \geq 0.7
$$

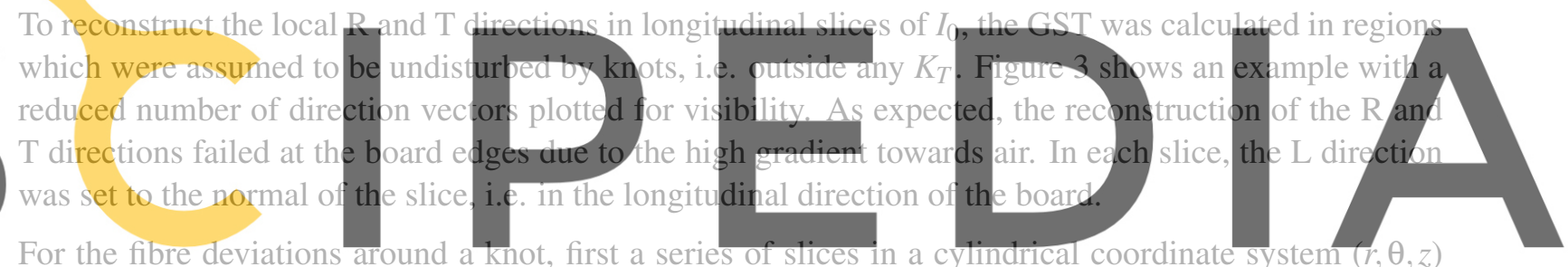

For the fibre deviations around a knot, first a series of slices in a cylindrical coordinate system $(r, \theta, z)$ was created, with the cylinder axis $z$ aligned with the pith and the $r$-z-plane aligned with the knot axis

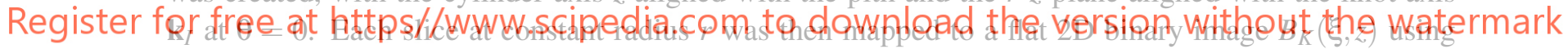

$\xi=r \theta$, where pixels of the section corresponding to the respective knot $K$ were set to 1 and all remaining pixels to 0 . An Euclidean distance map $[26] D_{K}(\xi, z)$ was calculated for $B_{K}$, where for each pixel, its distance to the closest pixel with value 1 was stored (see Figure 4a). After a Gaussian smoothing with $w_{\sigma_{3}}\left(\sigma_{3}=1\right)$, together with the lateral distance $\xi$, a flow field $F_{K}(\xi, z)$ was created according to equation 8. The isolines of $F_{K}(\xi, z)$ are exemplified in Figure $4 \mathrm{~b}$. The GST was calculated for $F_{K}(\xi, z)$ and the second eigenvector was used as the preliminary flow vector field $\mathbf{l}_{f}(\xi, z)$.

$$
F_{K}(\xi, z)=\left(w_{\sigma_{3}} * D_{K}(\xi, z)\right)^{\frac{1}{3}}|\xi|^{\frac{1}{10}}
$$

In a separate step, the pure diving vector field $\mathbf{l}_{d}(\xi, z)$ was constructed by spherical linear interpolation (Slerp) between the board axis (direction $\mathbf{z}$ ) and the knot axis $\mathbf{k}_{I}$, as a function of $D_{K}(\xi, z)$; for lower values of $D_{K}, \mathbf{l}_{d}(\xi, z)$ asymptotically approached $\mathbf{k}_{I}$, and for higher values it approached $\mathbf{z}$.

To combine the fields in the proximity of $D_{K}(\xi, z)=0$ and $\xi=0$, Slerp was used between $\mathbf{l}_{f}(\xi, z)$ and, depending on $\operatorname{argmin}\left(D_{K}(\xi, z), \xi\right)$, either $\mathbf{k}_{I}$ or $\mathbf{l}_{d}(\xi, z)$, respectively. As a result the flow field close to the knot diverged rather towards the knot axis in lateral locations, i.e. where $D_{K}(\xi, z)>\xi$, and it diverged 


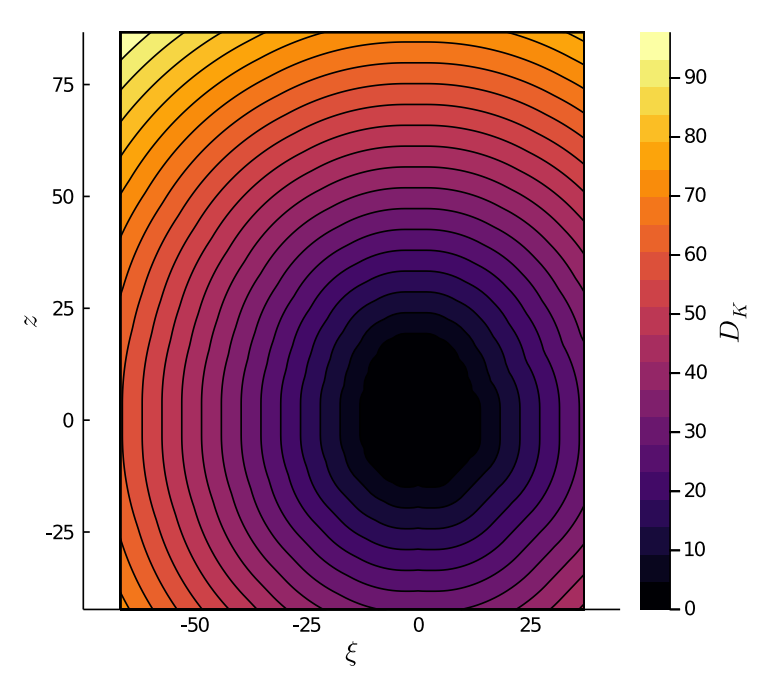

(a) Distance map of the knot

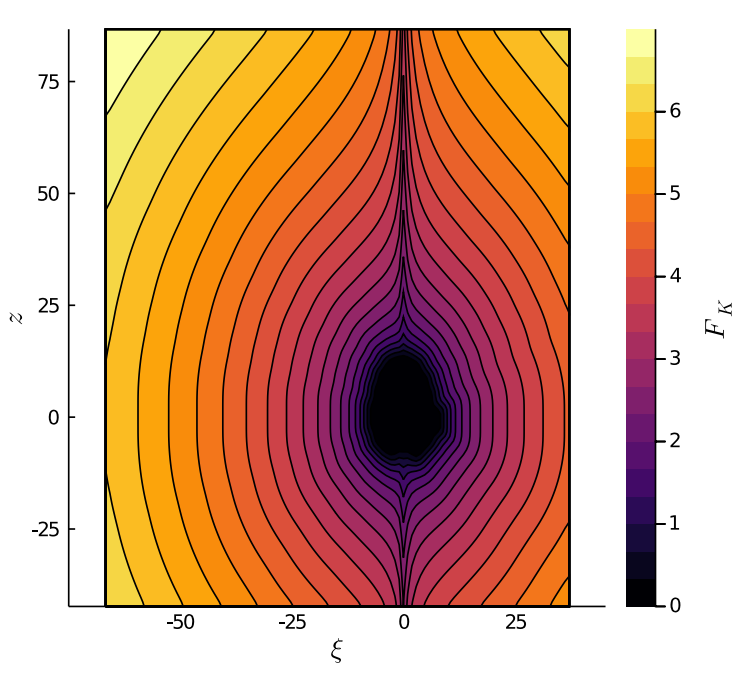

(b) Flow field $F_{K}$

Figure 4: Cylindrical slice around a knot

rather towards the dive field in vertical locations, where $D_{K}(\xi, z)<\xi$. A similar behaviour has been observed e.g. in [32].

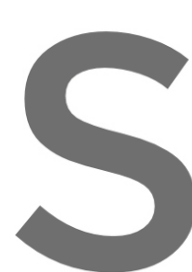

In a final step, the combir

direction z by Slerp, resul

$\mathrm{L}$ direction, the correspon

their cross product was used

Cartesian coordinates of the
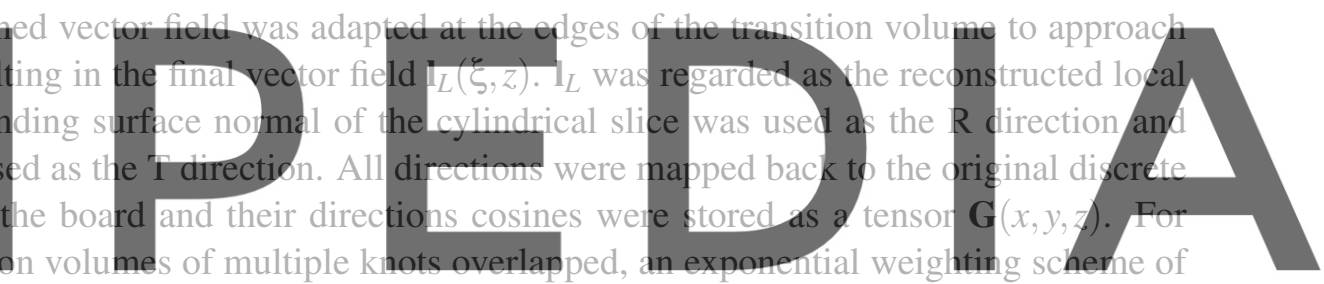

the directions, dependent on the distance to the respective knot, was applied like in [18]. Figures 5a

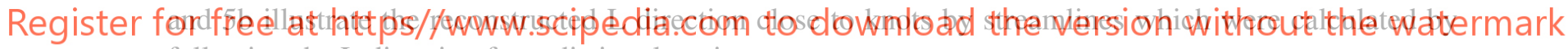
following the $\mathrm{L}$ direction from distinct locations.

\subsection{Finite element model}

A volume domain over the reconstructed geometry of the board was created and then meshed with quadratic tetrahedral elements using the programming interface of the free and open-source 3D meshing software Gmsh [33]. The representative mesh size was set $15 \mathrm{~mm}$ and the mesh was locally refined inside the transition volumes and knots to $3 \mathrm{~mm}$. The mesh was translated to an input file with C3D10 elements for Abaqus 2018 [34]. Figure 6 show an example of a meshed board.

Orthotropic material behaviour was assumed and the stiffness tensor $\mathbf{C}(x, y, z)$ in wood outside of knots was assumed to depend on the local density $\rho(x, y, z)$ which was calculated by linear interpolation from the discrete values in $I_{0}(x, y, z)$. A reference stiffness tensor $\mathbf{C}_{0}$ at $\rho_{0}=390 \mathrm{kgm}^{3}$ and $12 \% \mathrm{MC}$ from [10] was linearly scaled by the local density as in equation 9 . 


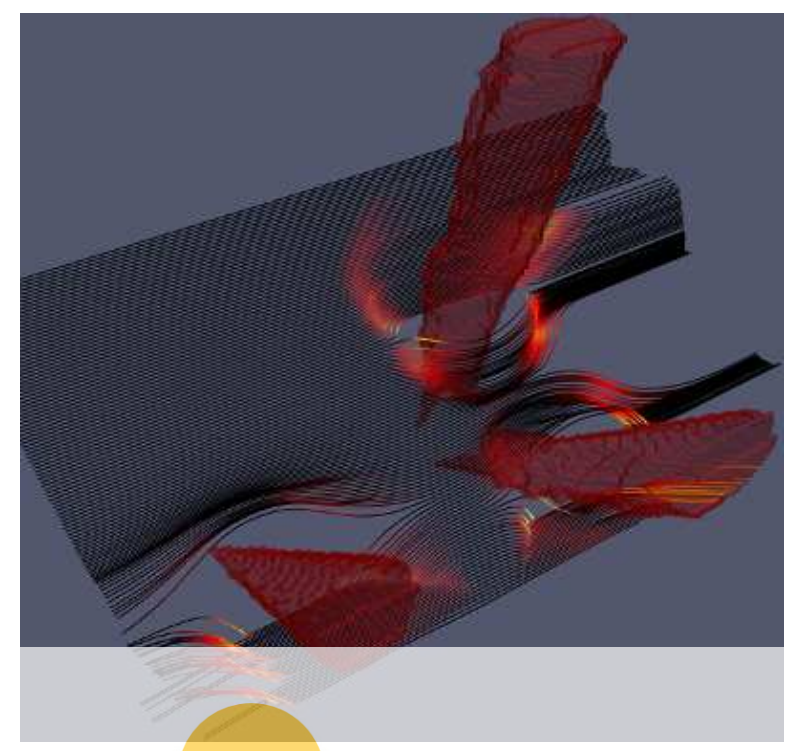

(a) Lateral locations around a knot group

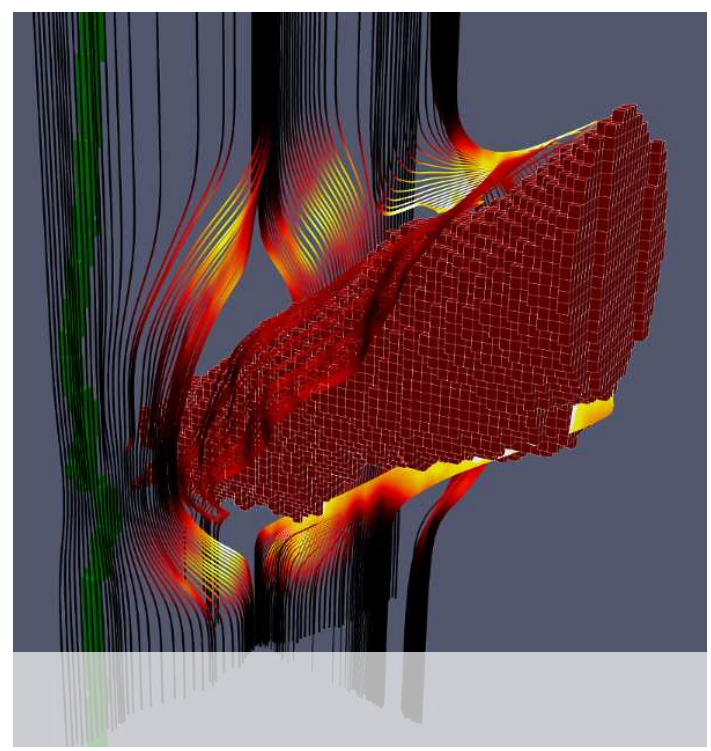

(b) Lateral and vertical locations around a knot

Figure 5: Streamlines following the L-direction; brighter colours indicate larger deviations
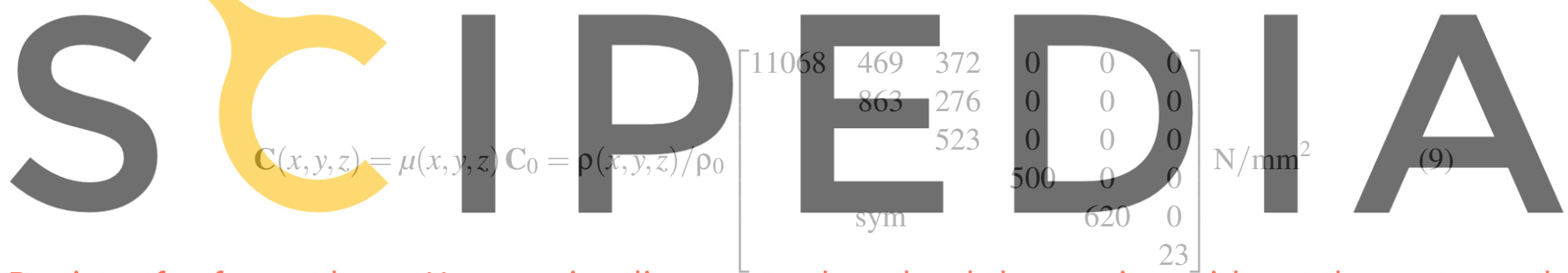

Register for free at https//www.scipedia.com to download the version without the watermark where the diagonal entries in $\mathbf{C}$ correspond in their order from top left to bottom right to the $\mathrm{L}, \mathrm{R}$ and $\mathrm{T}$ directions, and the L-R, L-T, and R-T shear planes. For material points inside the reconstructed knots, a slightly more isotropic stiffness tensor $\mathbf{C}_{K}$ was used using the same values as in [18]. The underlying local L-R-T coordinate system of $\mathbf{C}$ was similarly interpolated from the discrete reconstructed directions in $\mathbf{G}(x, y, z)$. For the mesh, $\mathbf{G}$ and $\mathbf{C}$, or respectively $\mathbf{C}_{K}$, were evaluated at the coordinates of each integration point (four per tetrahedron).

In Abaqus, each end surface of the board model was kinematically coupled to a reference point. One of the reference points was fixed in all translations and in rotation around $z$, and the other point was fixed only in translation along $x$ and $y$. The board model was loaded in pure bending in a linear static analysis by applying an equally large moment along $y$ at both reference points with opposed signs. The relative displacement $\delta$ corresponding to the experiments was extracted (see Figure 7). The momentdisplacement curve was compared to the experimental curve and to a linear regression line through the experimental data points. The relative error $e_{r}$ between the slope of the simulated and the regressed experimental curve was calculated. 


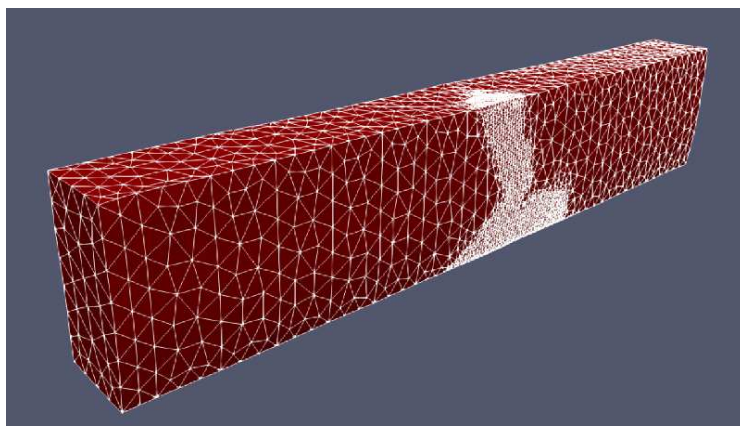

Figure 6: Board mesh with local refinements

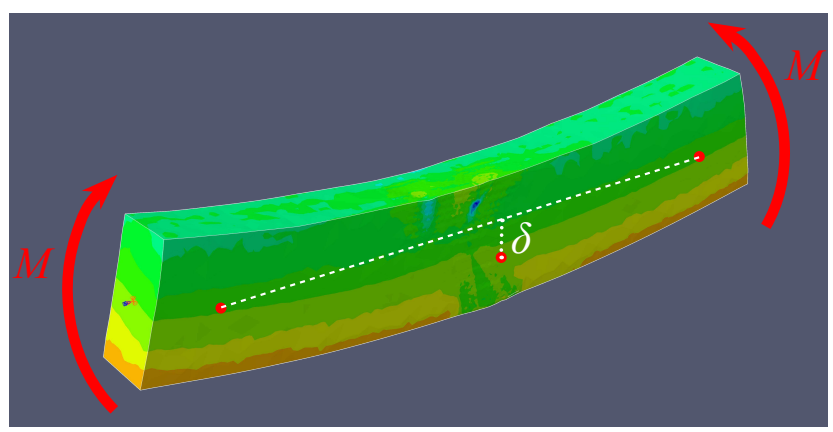

Figure 7: Stress plot of $\sigma_{L L}$ during pure bending

\section{RESULTS AND DISCUSSION}

The presented procedure is currently under development and calibration, however, some preliminary results exist. Figure 8 a shows the moment-displacement curves of the experiment, its regression line and the resulting curve of the simulation for one representative board. The relative error was $-2.31 \%$ as noted in the figure. For comparison, Figure $8 \mathrm{~b}$ shows the same curves, but for a simulation where the reconstructed fibre orientations have been ignored and the $\mathrm{L}$ direction was set along the board direction in the entire board. As expected, the simulation overestimated the stiffness of the board section in this case. A similar, consistent trend has been observed for other boards, when a comparison was made between accounting for the fibre

For boards where the procedure zones of stress concentration which failure regions of the experiments.
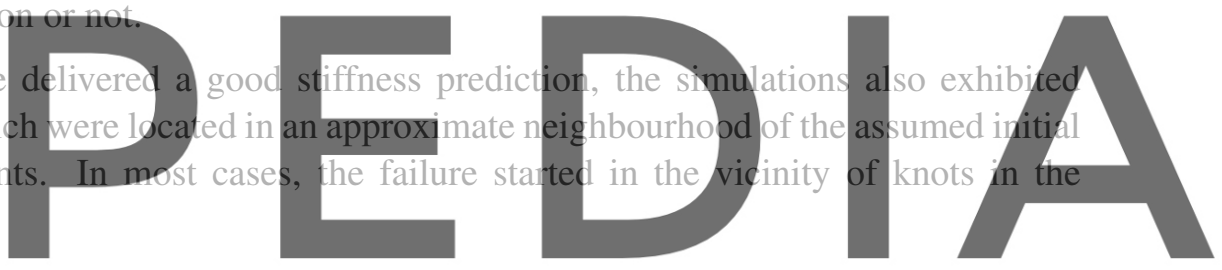

Is was observed that the stiffness of boards with a large number of small and dead knots was underes-

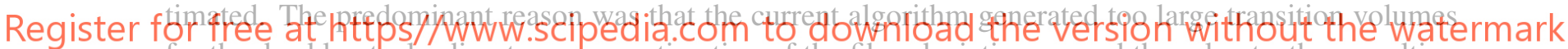
for the dead knots, leading to an overestimation of the fibre deviation around these knots, thus resulting in a less stiff board model.

\section{CONCLUSION AND OUTLOOK}

A procedure was presented to generate 3D FE models of timber boards based on CT scans. The boards were tested in four-point bending tests until failure and the local displacement in the pure bending zone was recorded. From the scans, the board, the knots and the pith were reconstructed using standard image processing tools by treating the stacked scans as a 3D image. A new procedure to reconstruct the fibre deviations around knots was used, which accounted for the gradient information in the image. A quadratic tetrahedral mesh was generated for the region of the board which was under pure bending in the tests. The fibre directions and a stiffness tensor scaled by the local density were transferred into each integration point of the mesh and the bending test was replicated. The moment-displacement curves of the experiments and the simulations were compared.

Preliminary results show that the procedure is able to realistically predict the experimentally observed 


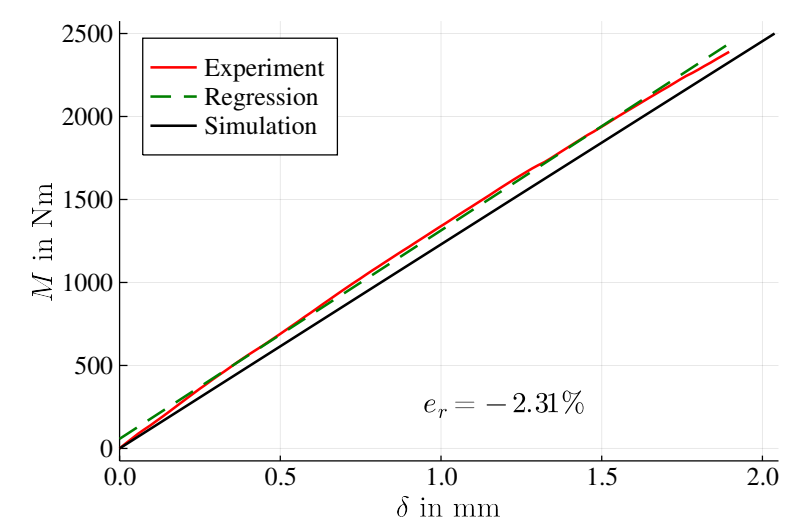

(a) accounting for the fibre deviations

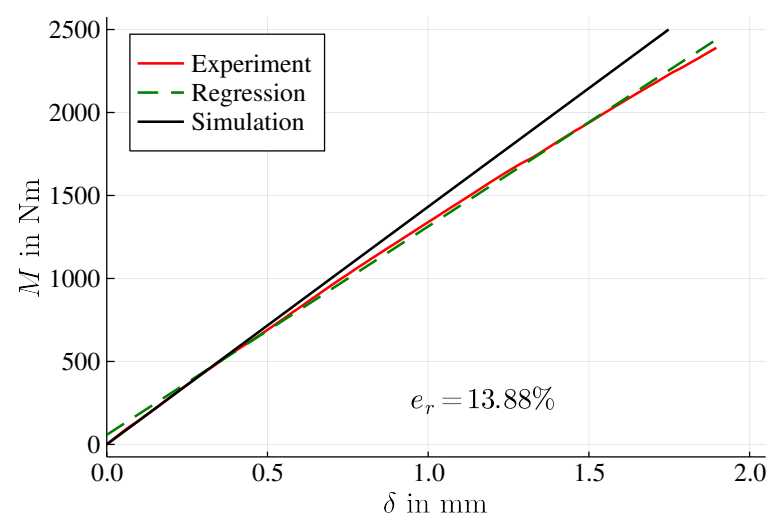

(b) not accounting for the fibre deviations

Figure 8: Moment-displacement curves of experiments and simulations for one board

local stiffness of the boards. Further development and calibration of the procedure is required to account for dead knots and their less pronounced fibre deviation zones. After calibration of the procedure to predict stiffness, it should be extended to indicate the region of failure initiation and the corresponding load. The results provide a first step towards enriching current timber grading methods by mechanical models. In the future, the procedure could be extended to indicate the stiffness and strength of virtual

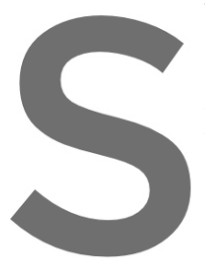
boards inside CT scanned

REFERENCES
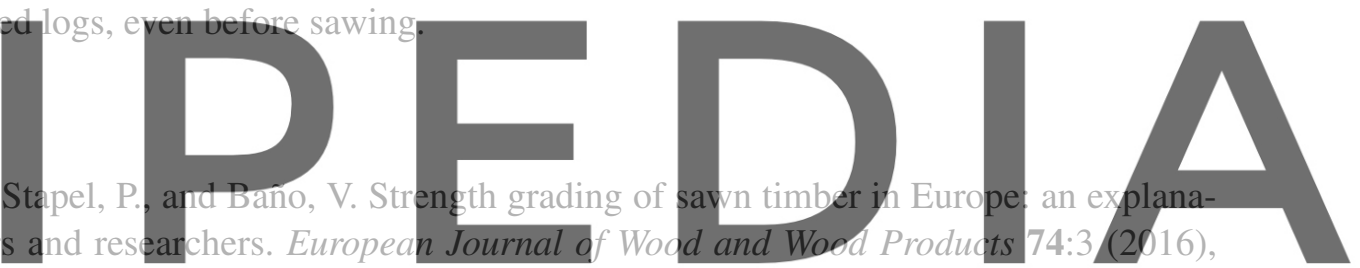

pp. 291-306. DOI: 10.1007/s00107-016-1034-1.

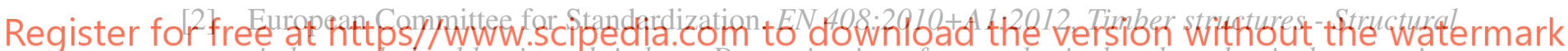
timber and glued laminated timber - Determination of some physical and mechanical properties. 2012.

[3] Hanhijärvi, A., Ranta-Maunus, A., and Turk, G. Potential of strength grading of timber with combined measurement techniques: Report of the Combigrade-project - phase 2. VTT Publications 686. 2008, pp. 3-81.

[4] Olsson, A. and Oscarsson, J. Strength grading on the basis of high resolution laser scanning and dynamic excitation: a full scale investigation of performance. European Journal of Wood and Wood Products 75:1 (2017), pp. 17-31. DOI: 10.1007/s00107-016-1102-6.

[5] Lindgren, O. Medical CT-Scanners for Non-Destructive Wood Density and Moisture Content Measurements. PhD thesis. Luleå University of Technology, 1992.

[6] Johansson, E. et al. Automated knot detection for high speed computed tomography on Pinus sylvestris L. and Picea abies (L.) Karst. using ellipse fitting in concentric surfaces. Computers and Electronics in Agriculture 96 (2013), pp. 238-245. DOI: 10.1016/ j . compag. 2013.06.003. 
[7] Longuetaud, F. et al. Automatic knot detection and measurements from X-ray CT images of wood: A review and validation of an improved algorithm on softwood samples. Computers and Electronics in Agriculture 85 (2012), pp. 77-89. DOI: 10.1016/j . compag . 2012 .03.013.

[8] Entacher, K. et al. Pith detection on CT-cross-section images of logs: An experimental comparison. 2008 3rd International Symposium on Communications, Control, and Signal Processing, ISCCSP 2008 March (2008), pp. 478-483. DOI: 10.1109/ISCCSP. 2008.4537273.

[9] Krähenbühl, A. et al. Knot detection in X-Ray CT images of wood. Lecture Notes in Computer Science (including subseries Lecture Notes in Artificial Intelligence and Lecture Notes in Bioinformatics) 7432 LNCS:PART 2 (2012), pp. 209-218. DOI: 10.1007/978-3-642-33191-6_21.

[10] Dinwoodie, J. M. Timber: Its nature and behaviour. 2nd ed. New York, NY: Taylor \& Francis, 2000.

[11] Lang, R. and Kaliske, M. Description of inhomogeneities in wooden structures: modelling of branches. Wood Science and Technology 47:5 (2013), pp. 1051-1070. DOI: 10.1007 / s00226013-0557-4.

[12] Gharib, M. et al. Three-dimensional constitutive modelling of arbitrarily orientated timber based on continuum damage mechanics. Finite Elements in Analysis and Design 135:March (2017), pp. 79-90. DOI: 10.1016/j.finel.2017.07.008.

[13] Lukacevic, M., Lederer, W., and Füssl, J. A microstructure-based multisurface failure criterion for the description of brittle and ductile failure mechanisms of clear-wood. Engineering Fracture Mechanics 176 (2017), pp. 83-99. DOI: 10.1016/j.engfracmech.2017.02.020.

[14] Schmidt, J. and Kaliske, M. Zur dreidimensionalen materialmodellierung von fichtenholz mittels eines mehrflächen-plastizitätsmodells. Holz als Roh - und Werkstoff 64:5 (2006), pp. 393-402. DOI: $10.1007 / \mathrm{s} 00107-006-0102-3$.

[15] $\mathrm{Hu}, \mathrm{M}$. et al. Local variation of modulus of elasticity in timber determined on the basis of noncontact deformation measurement and scanned fibre orientation. European Journal of Wood and Wood Products 73:1 (2015), pp. 17-27. DOI: 10.1007/s00107-014-0851-3.

[16] Jenkel, C. and Kaliske, M. Simulation of failure in timber with structural inhomogeneities using an automated FE analysis. Computers \& Structures 207 (2018), pp. 19-36. DOI: 10 . $1016 /$ j . compstruc.2017.11.016.

[17] $\mathrm{Hu}, \mathrm{M}$. et al. Modelling local bending stiffness based on fibre orientation in sawn timber. European Journal of Wood and Wood Products 76:6 (2018), pp. 1605-1621. DOI: 10.1007 /s00107-0181348-2.

[18] Lukacevic, M. et al. A 3D model for knots and related fiber deviations in sawn timber for prediction of mechanical properties of boards. Materials \& Design 166 (2019), p. 107617. DOI: 10.1016/j.matdes.2019.107617.

[19] Brancheriau, L. and Bailleres, H. Natural vibration analysis of clear wooden beams: a theoretical review. Wood Science and Technology 36:4 (2002), pp. 347-365. DOI: 10 . 1007 / s00226-0020143-7.

[20] Bezanson, J. et al. Julia: A Fresh Approach to Numerical Computing. SIAM Review 59:1 (2017), pp. 65-98. DOI: 10.1137/141000671.

[21] Canny, J. A Computational Approach to Edge Detection. IEEE Transactions on Pattern Analysis and Machine Intelligence PAMI-8:6 (1986), pp. 679-698. DOI: 10.1109/TPAMI . 1986. 4767851. 
[22] Illingworth, J. and Kittler, J. The Adaptive Hough Transform. IEEE Transactions on Pattern Analysis and Machine Intelligence PAMI-9:5 (1987), pp. 690-698. DOI: 10 . 1109 / TPAMI . 1987. 4767964.

[23] Foley, C. Modeling the Effects of Knots in Structural Timber. PhD thesis. Lund University, 2003.

[24] Guindos, P. Method for the integral calculation of the fiber orientation and the fundamental material properties of softwood logs and lumber. Holzforschung 70:10 (2016), pp. 981-991. DOI: $10.1515 / \mathrm{hf}-2015-0197$.

[25] Lukacevic, M. and Füssl, J. Numerical simulation tool for wooden boards with a physically based approach to identify structural failure. European Journal of Wood and Wood Products 72:4 (2014), pp. 497-508. DOI: 10.1007/s00107-014-0803-y.

[26] Ross, J. C. The Image Processing Handbook. 2nd ed. Boca Raton, FL: CRC Press, 1995.

[27] Bigun, J., Bigun, T., and Nilsson, K. Recognition by symmetry derivatives and the generalized structure tensor. IEEE Transactions on Pattern Analysis and Machine Intelligence 26:12 (2004), pp. 1590-1605. DOI: 10.1109/TPAMI.2004.126.

[28] Hansson, L., Couceiro, J., and Fjellner, B.-A. Estimation of shrinkage coefficients in radial and tangential directions from CT images. Wood Material Science \& Engineering 12:4 (2017), pp. 251256. DOI: $10.1080 / 17480272.2016 .1249405$.

[29] Krause, M. et al. Determination of the fibre orientation in composites using the structure tensor and local X-ray transform. Journal of Materials Science 45:4 (2010), pp. 888-896. DOI: $10.1007 /$ s10853-009-4016-4.

[30] Belyaev, A. Implicit Image Differentiation and Filtering with Applications to Image Sharpening. SIAM Journal on Imaging Sciences 6:1 (2013), pp. 660-679. DOI: 10.1137/12087092X.

[31] Bickley, W. G. Finite difference formulae for the square lattice. The Quarterly Journal of Mechanics and Applied Mathematics 1:1 (1948), pp. 35-42. DOI: 10.1093/qjmam/1.1.35.

[32] Hu, M. et al. Growth layer and fibre orientation around knots in Norway spruce: a laboratory investigation. Wood Science and Technology 52:1 (2018), pp. 7-27. DOI: 10.1007/s00226-0170952-3.

[33] Geuzaine, C. and Remacle, J.-F. Gmsh: A 3-D finite element mesh generator with built-in preand post-processing facilities. International Journal for Numerical Methods in Engineering 79:11 (2009), pp. 1309-1331. DOI: 10.1002/nme.2579.

[34] Dassault Systèmes Simulia Corp. Abaqus 2018 Documentation. Providence, RI, USA, 2017. 